\title{
Vitamin D and diabetes; a review
}

\author{
Liyanage PL GC ${ }^{1}$, Lekamwasam $\mathbf{S}^{2}$, Weerarathna $\mathbf{T P}^{2}$, Minipura $\mathbf{T}^{1}$ \\ ${ }^{\prime}$ Department of Pharmacology, ${ }^{2}$ Department of Medicine, Faculty of Medicine, University of Ruhuna, Galle, \\ Sri Lanka.
}

Correspondence: Dr.PL G C Liyanage

E-mail: gayanicl@yahoo.com

\begin{abstract}
Despite advanced therapeutic and primary prevention strategies, diabetes mellitus remains a significant global health care problem. Substantial morbidity and mortality associated with the disease, emphasize the importance of primary prevention. In Sri Lanka the prevalence of diabetes among male is $14.2 \%$ and $13.5 \%$ among females and these figures are expected to increase over the next few decades (1).
\end{abstract}

Vitamin D and its active metabolite $1,25 \mathrm{OH}_{2}$ cholecarciferol are widely known for their role in calcium homeostasis and bone metabolism. Recently, there has been a much evolving enthusiasm on the extra skeletal benefits of vitamin D. The basis for this appears to be related to the findings in several observational studies which reveal a close association between vitamin $\mathrm{D}$ and the development of autoimmune diseases, malignancies and metabolic abnormalities abnormalities such as type 2 diabetes (2).

The exact mechanism of vitamin D deficiency causing diabetes remains largely unknown. Vitamin D has a beneficial effect on insulin action either directly or indirectly by improving insulin exocytosis via activating calcium depending endopeptidases (3). It has been shown that type 1 diabetes is associated with an imbalance of pro anti-inflammatory cytokines and vitamin $\mathrm{D}$ acts as a potent immune suppressor and down regulator of transcription of proinflammatory cytokine genes, reducing the risk of type 1 diabetes (3).

This paper reviews the association of vitamin $\mathrm{D}$ with regard to the incidence, morbidity and mortality of diabetes.

\section{Incidence}

Several cross-sectional and cohort studies and randomized controlled trials have examined the association between vitamin $\mathrm{D}$ and the incidence of type 2 diabetes. A population based cross-sectional study reported a significant inverse association between serum $25(\mathrm{OH}) \mathrm{D} 3$ and the risk of developing diabetes. (OR 0.25, 95\% CI $0.11-0.60$ ) for non-Hispanic whites and OR 0.17, 95\% CI 0.08 - 0.37 for Mexican Americans (4).

Knekt et al.,observed that men with highest vitamin D quartile had a $82 \%$ lower risk of developing type 2 diabetes compared to men in the lowest vitamin D quartile. No significance association, however, was found among women (5). Nurses' Health Study by Pittas et al., showed that higher levels of plasma 25-OHD were associated with a lower risk for type 2 diabetes in women. (The relative OR between highest and lowest vitamin D quartiles was 0.52 , 95\% CI $0.33-0.83)(6)$.

Few studies such as the Nurses Health Study (7), the Women's Health Study (8) and the Japanese cohort study (9) have assessed the relationship between the dietary intake of vitamin $\mathrm{D}$ and the risk of developing diabetes. Of them, only the Japanese cohort study revealed a clear decreasing trend of type 2 diabetes with increasing dietary intake of vitamin D (9). The other two studies did not reveal any significant relationship between the total intake of vitamin D and incidence of type 2 diabetes.

Several interventional studies have studied the effects of vitamin D supplementation on the development of type 2 diabetes. Pittas et al, (2007) administered 700IU vitamin $\mathrm{D}_{3}$ in combination with calcium $500 \mathrm{mg}$ or placebo daily for 3 years for healthy adults and found that among participants 
with IFG those who took combined vitamin D and calcium supplements had a lower rise in fasting plasma glucose level $(\mathrm{p}=0.042)$ and a lower rise in HOMA-IR ( $\mathrm{p}=0.031)$ at 3 years compared to those on placebo. Butamong those who had normal fasting glycaemia there was no difference in the change of FPG and HOMA IR (4).

Von Hurst et al, (2009) showed a significant improvement in insulin sensitivity, fasting Insulin and insulin resistance with vitamin D supplementa-tion over placebo in non-diabetic, insulin resistant South Asian women after supplementation of 4000IU of vitamin $\mathrm{D}_{3}$ for a 6 months. (Insulin sensitivity increased $\mathrm{p}=0.01$, fasting insulin decreased $p=0.02$, Insulin resistance decreased $\mathrm{p}=0.03$ ) (10). Several other interventional studies carried out in participants with normal glycaemic level found no significant effect of vitamin D supplementation on fasting plasma glucose level and insulin resistance. (Niles L. 1984, de Bore et al, 2008, Avenell et al, 2009) (11-13).

Bin-Abbas et al and Janner $\mathrm{M}$ et al, found the prevalence of vitamin D deficiency among children and adolescent with diabetes to be high $(14,15)$. Further, Hypponen et al in their birth-cohort study showed a decreased frequency of type 1 diabetes with regular vitamin D supplementation. (OR 0.12, $95 \%, 0.03-0.51)$ and concluded that vitamin D supplementation in infants could help prevent or reverse the incidence of type 1 diabetes (16) and the EURODIAB Study also concluded that vitamin D supplementation in infancy is associated with a decreased risk of type 1 diabetes, (OR - 0.67 in $95 \%$ 0.53 - 0.86) (17).

Case-control studies done by Stene LC, et al (2000 and 2003) found that when mothers consumed cod liver oil, (a known vitamin D supplement) during pregnancy their offspring had a lower risk of diabetes (OR - 0.30 in $95 \%$ CI 0.12 to 0.75$)(18,19)$. Also the use of cod liver oil during the first year of life may reduce the risk of type 1 diabetes. (OR - 0.74 in $95 \%$ CI 0.56 to 0.99$)(20)$.

\section{Complications}

Although the association between vitamin D and diabetes has been studied in several observational and interventional studies, evidence for the association between complications of diabetes and vitamin $\mathrm{D}$ are sparse. A cross-sectional study carried out by Massimo C et al, (2005) found that the prevalence of cardiovascular disease among diabetics is greater when they have co-exsistant hypovitaminosis D (Odds ratio 1.70 in 95\%, CI 1.1-2.6, p-0.01) (21). Furthermore, two randomized controlled trials by Sugden JA et al, (2007 and 2010) showed a significant improvement in Flow mediated vasodilation (a tool of assessing endothelial function) in the vitamin D received group compared to the placebo group $(22,23)$. Measurement of endothelial function is a surrogate marker to assess cardiovascular risk, especially atherosclerosis (24).

Another study showed a clinically and statistically significant reduction in systolic blood pressure among vitamins D supplemented group than in the placebo group after 8 weeks of therapy (25). No significant changes were found, however, with vitamin D supplementation on HbA1c or insulin resistance even with higher doses of vitamin D3 (26).

Few studies have assessed the association of vitamin D with diabetic neuropthy. Paul Lee et al in 2010 showed that patients with type 2 diabetes who have low vitamin D levels had distressing neuropathic pain according to a visual analogue scale (VAS) and pain scores for both the VAS $\left(r^{2}=0.10\right)$ and McGill pain questionnaire (MPQ $r^{2}=0.18$ ). Repletion of vitamin D with mean oral daily dose of 2059IU for 3 months resulted in a significant reduction in pain scores on both the VAS and MPQ, $-48.5 \%$ and $-39.4 \%$, respectively (27). A similar study by Soderstrom L. Hrevealed that vitamin D insufficiency is associated with the self-reported peripheral neuropathy even after adjusting for demographic factors, medications and diabetes duration (28). Shehab D et al, also showed that vitamin $\mathrm{D}$ deficiency is an independent risk factor of diabetic neuropathy among patients with established diabetes. A greater proportion $(81.5 \%)$ of patients with diabetic neuropathy had vitamin D deficiency compared to patients without neuropathy $(60.4 \%)$ and diabetic peripheral neuropathy associated with vitamin D deficiency. (OR - 3.47: CI 1.04 - 11.56, $\mathrm{p}=0.043)$ (29). 


\section{Nephropathy}

The potential relationship between thevitamin D and diabetic retinopathy has been the focus in several cross sectional studies. In 2000, Aksoy H et al reported that mean 1, $25\left(\mathrm{OH}_{2}\right) \mathrm{D} 3$ concentration fell with increasing severity of diabetic retinopathy. (DR) (background DR $63.4 \pm 17.26 \mathrm{pmol} / \mathrm{L}$, pre-proliferative DR $47.7 \pm 13.27 \mathrm{pmol} / \mathrm{L}$ and for proliferative DR $43.1 \pm 19.45 \mathrm{pmol} / \mathrm{L})$. They also found that, compared to the control group, serum $25(\mathrm{OH}) \mathrm{D}$ concentration was lower in diabetic patients (30). A similar study by Patricia A et al, also reported the same results which the percentage of individuals with vitamin D deficiency increased with the severity of retinopathy. But the regression analysis of retinopathy severity vs serum 25hydroxyvitamin D did not reveal a statistically significant relationship between the two variables (31). Alam et al, evaluated the relationship between the two variables and found that there was no difference in serum 25-hydroxyvitamin D concentration between diabetic retinopathy group and the diabetic non-retinopathy group (32). Although most of the studies showed a increased vitamin D deficiency with the severity of the diabetic retinopathy, none of the studies have shown a statistically significant difference between the two variables.

\section{Vitamin D and mortality in Diabetes}

Emerging evidence suggest that inadequate vitamin D may influence the mortality in chronic diseases, especially diabetes. Christel et al, followed up 227 patients with type 1 diabetes and during the follow up 44 (18\%) patients died, 81 (37\%) patients developed microalbuminuria, 27 (12\%) developed macroalbuminuria and furthermore 192 (87\%) patients developed retinopathy. The hazard ratio of mortality in subjects with severe vitamin D deficiency was 2.7 (95\% CI : 1.1 to 6.7$)$. They concluded that severe vitamin $\mathrm{D}$ deficiency independently predicts all causes of mortality in patients with type 1 diabetes but not the development of microvascular complications (33). Cristel J et al, showed increased all cause mortality among patients with severe vitamin D deficiency during the follow up of 289 patients with type 2 diabetes. (Hazard ratio was 1.96 with $95 \%$ CI 1.29-2.98) (34).

\section{Summary}

Previous studies have shown a significant association between serum vitamin D status and the incidence of type 2 diabetes. But the dietary vitamin $\mathrm{D}$ intake did not show a significant reduction of diabetes incidence. However, in clinical trials, vitamin D supplementation showed a marked improvement of insulin sensitivity and fasting insulin levels in people with insulin resistance but not in people with normal glucose tolerance. However few case control studies have shown that vitamin D supplementation during pregnancy and first year of life could reduce the incidence of type 1 diabetes.

Intervetional studies showed an association between cardiovascular disease and hypovitaminosis D. Few clinical trials also reported a significant improvement of endothelial function resulting in lower systolic blood pressure with vitamin D supplementation in type 2 diabetes.

Vitamin D therapy probably exerts beneficial effects on diabetic microvascular complications as well. Several cross sectional and longitudinal studies have reported an inverse association between serum vitamin D3 level and neuropathic pain and the severity of diabetic retinopathy. Studies have reported that severe vitamin D deficiency is associated with increased mortality in patients with both types of diabetes. Considering above results a hypothesis can be developed that vitamin D status is a significant determinant of the incidence, occurrence of complications, and the all cause mortality of diabetes.

\section{References}

1. Katulanda P, Sheriff MHR, Matthews DR. The diabetes epidemic in Sri Lanka a growing problem. Ceylon Medical Journal, 2006; 51(1): 26-8.

2. Visweswaran R Kasi, H Lekha. Extra skeletal effects and manifestations of Vitamin D deficiency. Indian Journal of Endocrinology and Metabolism, 2013;17(4): 602.

3. Seshadri Krishna G, Bubblu Tamilselvan, and Amarabalan Rajendran. Role of vitamin D in diabetes. Journal of Endocrinology and Metabolism, 2011;1(2): 47-56. 
4. Pittas Anastassios G, et al. The effects of calcium and vitamin D supplementation on blood glucose and markers of inflammation in nondiabetic adults. Diabetes Care, 2007; 30(4): 980-6.

5. Knekt Paul, et al. Serum vitamin D and subsequent occurrence of type 2 diabetes. Epidemiology, 2008; 19(5): 666-71.

6. Pittas Anastassios G, et al. Plasma 25-hydroxyvitamin D concentration and risk of incident type 2 diabetes in women. Diabetes Care, 2010;33(9): 2021-3.

7. Pittas Anastassios G, et al. Vitamin D and calcium intake in relation to type 2 diabetes in women. Diabetes Care, 2006; 29(3): 650-6.

8. Liu Simin, et al. Dietary calcium, vitamin $\mathrm{D}$, and the prevalence of metabolic syndrome in middle-aged and older US women. Diabetes Care, 2005; 28(12): 2926-32.

9. Kirii $\mathrm{K}$, et al. Calcium, vitamin $\mathrm{D}$ and dairy intake in relation to type 2 diabetes risk in a Japanese cohort. Diabetologia, 2009; 52(12): 2542-50

10. von Hurst, Pamela R., Welma Stonehouse, Jane Coad. Vitamin D supplementation reduces insulin resistance in South Asian women living in New Zealand who are insulin resistant and vitamin D deficient - a randomised, placebocontrolled trial. British Journal of Nutrition, 2010; 103(4): 549-55.

11. Nilas L, Christiansen C. Treatment with vitamin D or its analogues does not change body weight or blood glucose level in postmenopausal women. International Journal of Obesity, 1983;8(5): 407-11.

12. De Boer, Ian $\mathrm{H}$, et al. Calcium plus vitamin D supplementation and the risk of incident diabetes in the Women's Health Initiative. Diabetes Care, 2008; 31(4): 701-7.

13. Avenell Alison, et al. Vitamin D supplementation and type 2 diabetes: a substudy of a randomised placebo-controlled trial in older people (RECORD trial, ISRCTN 51647438). Age and Ageing, 2009; afp109.

14. Bin-Abbas, Bassam S, et al. Vitamin D levels in Saudi children with type 1 diabetes. Saudi Medical Journal, 2011; 32(6): 589-92.

15. Janner Marco, et al. High prevalence of vitamin D deficiency in children and adolescents with type 1 diabetes. Swiss Med Wkly, 2010; 140: w13091.
16. Hyppönen Elina, et al. Intake of vitamin D and risk of type 1 diabetes: a birth-cohort study. The Lancet, 2001; 358(9292): 1500-3.

17. Levy-Marchal C, Patterson CC, Green A. Geographical variation of presentation at diagnosis of type I diabetes in children: The EURODIAB study. Diabetologia, 2001; 44(3): B75-B80.

18. Stene LC, et al. Use of cod liver oil during pregnancy associated with lower risk of Type I diabetes in the offspring. Diabetologia, 2000; 43(9): 1093-8.

19. Stene Lars C, Geir Joner. Use of cod liver oil during the first year of life is associated with lower risk of childhoodonset type 1 diabetes: a large, population-based, case-control study. The American Journal of Clinical Nutrition, 2003; 78(6): 1128-34.

20. Zipitis Christos S, Anthony K Akobeng. Vitamin D supplementation in early childhood and risk of type 1 diabetes: a systematic review and meta-analysis. Archives of Disease in Childhood, 2008;93(6): 512-7.

21. Cigolini Massimo, et al. Serum 25-hydroxyvitamin D3 concentrations and prevalence of cardiovascular disease among type 2 diabetic patients. Diabetes Care, 2006; 29(3): $722-4$.

22. Giovannucci, Edward, et al. 25-hydroxyvitamin D and risk of myocardial infarction in men: a prospective study. Archives of Internal Medicine, 2008; 168(11): 1174-80.

23. Sugden J A, et al. Vitamin D improves endothelial function in patients with Type 2 diabetes mellitus and low vitamin D levels. Diabetic Medicine, 2008; 25(3); 320-5.

24. Witham MD, et al. The effect of different doses of vitamin D3 on markers of vascular health in patients with type 2 diabetes: A randomised controlled trial. Diabetologia, 2010; 53(10): 2112-9.

25. Widlansky Michael E, et al. The clinical implications of endothelial dysfunction. Journal of the American College of Cardiology,42.7 (2003): 1149-1160.

26. Witham MD, et al. The effect of different doses of vitamin D3 on markers of vascular health in patients with type 2 diabetes: A randomised controlled trial. Diabetologia, 2010; 53(10): 2112-9.

27. Lee Paul, Roger Chen. Vitamin D as an analgesic for patients with type 2 diabetes and neuropathic pain. Archives of Internal Medicine, 2008; 168(7): 771-2. 
28. Soderstrom LH, et al. Association between vitamin D and diabetic neuropathy in a nationally representative sample: Results from 20012004 NHANES. Diabetic Medicine, 2012; 29(1): 50-5.

29. Shehab D, et al. Does Vitamin D deficiency play a role in peripheral neuropathy in Type 2 diabetes? Diabetic Medicine, 2012; 29(1): 43-9.

30. Aksoy Hülya, et al. Serum 1, 25 dihydroxy vitamin D $(1,25(\mathrm{OH})<$ sub $>2</$ sub $>$ D $<$ sub $>3</$ sub $>), 25$ hydroxy vitamin D (25 (OH) D) and parathormone levels in diabetic retinopathy. Clinical Biochemistry, 2000; 33(1): 47-51.

31. Patrick Patricia A, et al. Vitamin d and retinopathy in adults with diabetes mellitus. Archives of Ophthalmology, 2012; 130(6): 756-60.
32. Alam Uazman. Nine Chapter IX-Vitamin D Deficiency is not associated with Diabetic Retinopathy or Maculopathy: A Cross Sectional study. Vitamin $D$ and Diabetic Neuropathy, 300.

33. Joergensen Christel, et al. Vitamin D levels, microvascular complications, and mortality in type 1 diabetes. Diabetes Care, 2011;34(5): 1081-5.

34. Joergensen Christel, et al. Vitamin D levels and mortality in type 2 diabetes. Diabetes Care, 2010; 33(10): 2238-43. 